\title{
Hyperhomocysteinemia is a risk factor for colorectal adenoma in women
}

\author{
Yun Jeong Lim, ${ }^{1}$ Jeong Hwan Kim, ${ }^{2}$ Sue K. Park, ${ }^{3}$ Hee Jung Son, ${ }^{4}$ Jae J. Kim ${ }^{4}$ and Young-Ho Kim ${ }^{4, *}$ \\ 'Department of Internal Medicine, Dongguk University Ilsan Hospital, Dongguk University College of Medicine, Goyang, Korea \\ 2Department of Internal Medicine, Konkuk University Hospital, Konkuk University School of Medicine, Seoul, Korea \\ ${ }^{3}$ Department of Preventive Medicine, SNU College of Medicine, Seoul, Korea \\ ${ }^{4}$ Department of Internal Medicine, Samsung Medical Center, Sungkyunkwan University School of Medicine, Irwon-Dong 50, Gangnam-gu, Seoul 135-710, Korea
}

(Received 24 November, 2011; Accepted 7 January, 2012; Published online 11 May, 2012)

Homocysteine is involved in a one-carbon transfer reaction, which is important for DNA synthesis and methylation. High level of plasma homocysteine, biochemical marker of folate status, is known to be a risk factor for cancer. However, it is inconclusive as to whether plasma homocysteine concentration can predict colorectal adenoma. We conducted a case-control study to determine whether hyperhomocysteinemia is a risk factor for adenoma. Data from 1,039 subjects who underwent a colonoscopy and plasma homocysteine concentration determination during health examinations at single center over a two-year period were analyzed. The subjects were classified into two groups (422 adenoma and 617 controls). Subjects defined as having advanced adenomas were those with more than three adenomas, over $1 \mathrm{~cm}$ in size, high grade dysplasia, or villous components. Male, old age, high body mass index, low high-density lipoprotein-cholesterol, smoking, fasting glucose, and past history of colon polyps were significantly associated with adenoma according to multiple logistic regression. According to subgroup analysis by gender, plasma homocysteine concentration was not associated with adenoma in males; however, a high plasma homocysteine concentration significantly increased the risk of adenoma as well as advanced adenoma in females. Hyperhomocysteinemia is a risk factor for colorectal adenoma in women.

Key Words: risk factors, homocysteine, adenoma

Colorectal cancer is the third most common malignancy and the second leading cause of cancer mortality in the United States. ${ }^{(1)}$ Most sporadic colorectal cancers arise from preexisting adenomas. ${ }^{(2)}$ Recently, a marked increment in colorectal cancer as well as adenoma was documented in Korea as a result of Western influences including high fat and low vegetable consumption along with sedentary lifestyles. Understanding risk factors for colorectal cancer and adenoma may guide the development of strategies targeted towards its prevention. Recently, many data have suggested that cardiovascular risk factors, for examples, metabolic syndrome, and/or obesity, are clustered with risk factors for colorectal adenoma or cancer. ${ }^{(3-9)}$ Plasma homocysteine, a biochemical marker of folate status, is a well-known risk factor for atherosclerotic vascular disease and cancer. ${ }^{(10-13)}$ However, it has not been determined in a large study population as to whether folate deficiency, demonstrated by a high plasma homocysteine concentration can increase the risk of colorectal adenoma.

The purpose of this study was to determine whether plasma homocysteine level, a cardiovascular biomarker, is a risk factor for the adenoma, a precursor of colorectal cancer.

\section{Materials and Methods}

Subjects. A total of 1,039 patients who had screening colonoscopies and plasma homocysteine levels measured at the same time over a two-year period at the Samsung Medical Center were enrolled in this investigation. The subjects had completed a clinical survey to determine their eligibility. The following criteria were considered as grounds for exclusion: a history of colon cancer, prior colonic surgery, inflammatory bowel disease, severe liver, lung, renal, hematological or connective tissue disorders, and other malignancies. The 1,039 subjects were classified into two groups according to their premalignant potential; the case group had histologically confirmed colorectal adenomas, including tubular, villous adenomas, or serrated adenomas, and the control group had normal colonoscopy findings, non-polyp benign lesions such as nonspecific inflammation or histologically confirmed hyperplastic polyps (Fig. 1). This study was approved by the Institutional Review Board of Samsung Medical Center.

Laboratory assay and measurements. Plasma homocysteine concentration was measured by high performance liquid chromatography with a fluorescence detector (HP 1100, Agilent Technologies, MN). Colonoscopic examination was performed up to the cecal end or terminal ileum after bowel preparation with polyethylene glycol. Advanced adenoma was defined as an estimated diameter $\geq 10 \mathrm{~mm}$ (the largest size was used for multiple adenomas), containing $>25 \%$ villous features, high grade dysplasia, or $>$ three incidences of adenoma.

Body Mass Index (BMI) was calculated as weight (kilograms) divided by height squared (square meters). Data including smoking, alcohol consumption, diabetes mellitus, hypertension, and past history of colon polyps were collected from a standardized questionnaire using the gathered magnetic entry method. Heavy drinking was defined as drinking $>80 \mathrm{~g}$ of alcohol a day.

Statistics. The chi-squared test and independent $t$ test were used for comparison of the discrete variables (gender, smoking, alcohol, diabetes, hypertension, and past history of colon polyps) and continuous variables (age, BMI, lipid profiles, fasting glucose, homocysteine), respectively. The continuous variables measured in this study were expressed as the mean \pm SD. Age, gender, BMI, triglyceride, high-density lipoprotein-cholesterol (HDL-C), homocysteine, fasting glucose, smoking, alcohol, diabetes, hypertension, and past history of colon polyps were adjusted as independent variables in the multiple logistic regression model. Multiple logistic regression analysis by gender-subgroup was also performed to evaluate the association between plasma homocysteine concentration and adenoma. For each variable, the odds

*To whom correspondence should be addressed.

E-mail: younghokim@skku.edu 
A

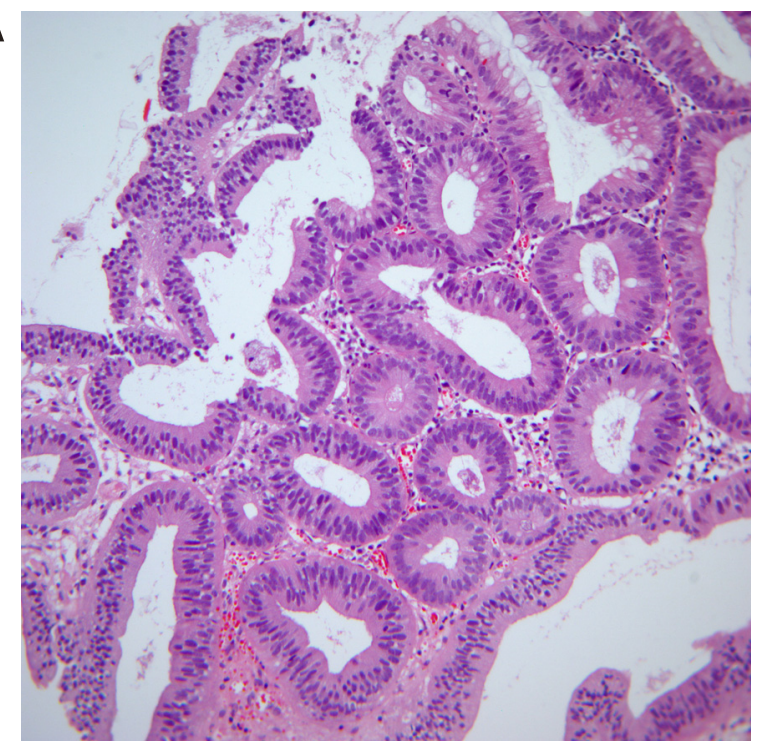

B

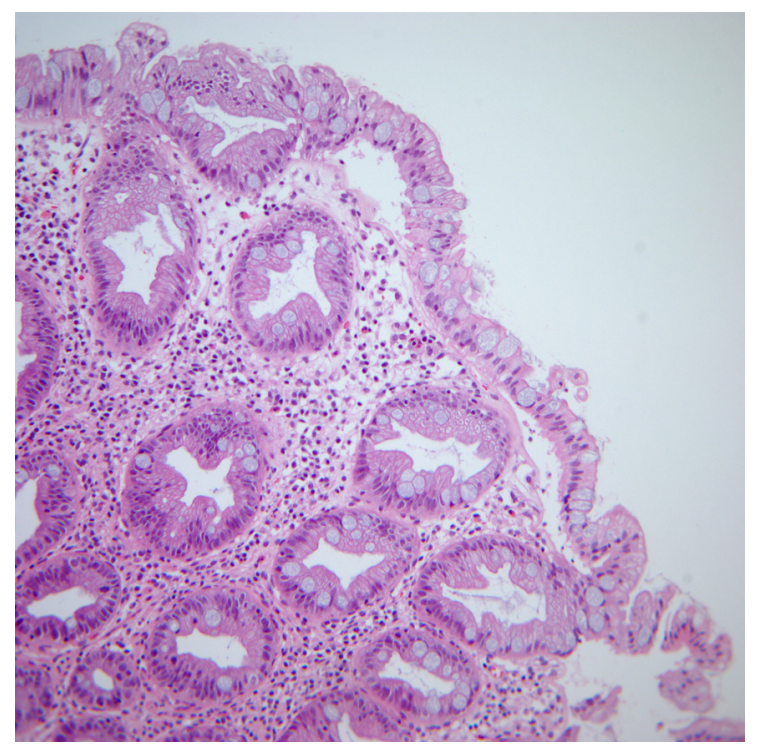

Fig. 1. Pathologic features of tubular adenoma (A), hyperplastic polyp (B). (A) Tubular adenoma showing low grade dysplasia (H\&E, $\times 200)$. (B) Hyperplastic polyp showing hyperplastic epithelia with stellate lumens $(H \& E, \times 200)$.

Table 1. Risk tactors tor adenoma in the study population

\begin{tabular}{|c|c|c|c|}
\hline & Control $(n=617)$ & Adenoma $(n=422)$ & $p$ value \\
\hline Age (year) & $49.41 \pm 8.07$ & $52.18 \pm 7.04$ & $<0.001$ \\
\hline Male gender & 499 (80.87\%) & $376(89.1 \%)$ & 0.0004 \\
\hline $\mathrm{BMI} *\left(\mathrm{~kg} / \mathrm{m}^{2}\right)$ & $24.45 \pm 2.75$ & $25.08 \pm 2.81$ & 0.0004 \\
\hline Total cholesterol (mg/dl) & $195.50 \pm 32.59$ & $198.58 \pm 30.30$ & 0.1303 \\
\hline Triglyceride (mg/dl) & $135.90 \pm 79.68$ & $156.52 \pm 89.90$ & 0.0002 \\
\hline $\mathrm{HDL}-\mathrm{C}^{\dagger}(\mathrm{mg} / \mathrm{dl})$ & $53.82 \pm 12.82$ & $51.12 \pm 12.72$ & 0.0009 \\
\hline LDL-C $C^{\ddagger}(\mathrm{mg} / \mathrm{d} 1)$ & $129.57 \pm 29.35$ & $132.92 \pm 28.70$ & 0.0691 \\
\hline Fasting glucose & $93.59 \pm 17.22$ & $97.20 \pm 21.29$ & 0.0086 \\
\hline Smoking & 349 (56.56\%) & $298(70.62 \%)$ & $<0.001$ \\
\hline Heavy alcohol consumption & $55(8.91 \%)$ & $24(5.69 \%)$ & 0.0589 \\
\hline Homocysteine $(\mu \mathrm{g} / \mathrm{l})$ & $13.16 \pm 5.88$ & $13.29 \pm 3.90$ & 0.562 \\
\hline Diabetes & $88(14.26 \%)$ & $89(21.09 \%)$ & 0.0152 \\
\hline Hypertension & $121(19.61 \%)$ & $86(20.38 \%)$ & 0.7608 \\
\hline Past history of colon polyps & $86(13.94 \%)$ & $99(23.46 \%)$ & $<0.0001$ \\
\hline
\end{tabular}

ratio (OR) and 95\% confidence interval $(\mathrm{CI})$ were determined. All analyses were performed with SAS ver. 9.2 (SAS Institute Inc., Cary, NC).

\section{Results}

Risk factors for adenoma. The prevalence of adenoma in the study population was $40.6 \%(422 / 1,039)$. Demographical, anthrometrical, and clinical and laboratory factors between the control and adenoma groups were univariately and multivariately analyzed (Table 1 and 2). Old age, male gender, high BMI, high triglycerides, low HDL-C level, elevated fasting-glucose, smoking, diabetes, and past colon polyp history were associated with adenoma in univariate analysis. According to multiple logistic regression, adenoma developed more with aging and male gender was a strong risk factor for adenoma. Incidence of adenoma increased along with BMI and a low HDL-C level was significantly associated with adenoma. Adenoma was found more
Table 2. Multiple logistic regression model

\begin{tabular}{lcr}
\hline Risk factor & $\begin{array}{c}\text { Odds ratio }(95 \% \\
\text { confidence interval) }\end{array}$ & $p$ value \\
\hline Age (years) & $1.041(1.032-1.051)$ & $<0.0001$ \\
Female gender & $0.584(0.464-0.735)$ & $<0.0001$ \\
Smoking & $1.633(1.369-1.948)$ & $<0.0001$ \\
Heavy alcohol consumption & $0.827(0.612-1.117)$ & 0.2152 \\
Diabetes & $1.165(0.851-1.596)$ & 0.3401 \\
Hypertension & $0.872(0.722-1.054)$ & 0.1561 \\
Colon polyp history & $1.446(1.206-1.735)$ & $<0.0001$ \\
BMI* & $1.043(1.012-1.075)$ & 0.0065 \\
Triglyceride & $1.002(0.999-1.004)$ & 0.1572 \\
Fasting glucose & $1.002(0.997-1.006)$ & 0.4722 \\
HDL-C & $0.994(0.988-1.000)$ & 0.0393 \\
\hline
\end{tabular}

*BMI; body mass index, ${ }^{+} \mathrm{HDL}-\mathrm{C}$; high-density lipoprotein-cholesterol. 
Table 3. Homocysteine level as a risk factor for colorectal adenomatous polyp

\begin{tabular}{|c|c|c|c|c|c|}
\hline & $\begin{array}{c}\text { Number of } \\
\text { controls }\end{array}$ & $\begin{array}{l}\text { Number of } \\
\text { adenomatous } \\
\text { polyp cases }\end{array}$ & OR $(95 \% \mathrm{Cl})$ & $\begin{array}{c}\text { Number of } \\
\text { advanced } \\
\text { adenomatous } \\
\text { polyp cases }\end{array}$ & OR $(95 \% \mathrm{Cl})$ \\
\hline Total* & 617 & 422 & & 313 & \\
\hline Homocysteine per 1 mg/l & & & $0.99(0.97-1.02)$ & & $1.00(0.96-1.02)$ \\
\hline \multicolumn{6}{|l|}{ Homocysteine } \\
\hline$-9 \quad \mu g / l$ & 97 & 33 & 1 & 24 & 1 \\
\hline $10-11$ & 137 & 96 & $1.39(0.85-2.29)$ & 71 & $1.51(0.87-2.64)$ \\
\hline $12-13$ & 171 & 132 & $1.35(0.84-2.18)$ & 95 & $1.41(0.83-2.41)$ \\
\hline $14-17$ & 158 & 123 & $1.28(0.79-2.08)$ & 100 & $1.55(0.90-2.67)$ \\
\hline \multirow[t]{2}{*}{$18+$} & 54 & 38 & $1.19(0.65-2.16)$ & 23 & $1.03(0.51-2.07)$ \\
\hline & & & $p$-trend $=0.387$ & & $p$-trend $=0.345$ \\
\hline Males** & 499 & 376 & & 279 & \\
\hline Homocysteine per $1 \mu \mathrm{g} / \mathrm{l}$ & & & $0.98(0.95-1.01)$ & & $1.03(0.76-1.39)$ \\
\hline \multicolumn{6}{|l|}{ Homocysteine } \\
\hline$-9 \quad \mu g / l$ & 37 & 22 & 1 & 18 & 1 \\
\hline $10-11$ & 102 & 77 & $1.13(0.61-2.11)$ & 57 & $1.08(0.55-2.11)$ \\
\hline $12-13$ & 154 & 121 & $1.15(0.63-2.08)$ & 86 & $1.04(0.55-1.97)$ \\
\hline $14-17$ & 153 & 119 & $1.13(0.62-2.05)$ & 96 & $1.18(0.62-2.24)$ \\
\hline \multirow[t]{2}{*}{$18+$} & 53 & 37 & $1.06(0.53-2.12)$ & 22 & $1.79(0.36-1.72)$ \\
\hline & & & $p$-trend $=0.975$ & & $p$-trend $=0.908$ \\
\hline Females** & 118 & 46 & & 34 & \\
\hline Homocysteine per $1 \mu \mathrm{g} / 1$ & & & $1.21(1.04-1.41)$ & & $1.30(1.09-1.55)$ \\
\hline \multicolumn{6}{|l|}{ Homocysteine } \\
\hline$-9 \quad \mu g / 1$ & 60 & 11 & 1 & 6 & 1 \\
\hline $10-11$ & 35 & 19 & $2.60(1.09-6.18)$ & 14 & $3.78(1.30-10.98)$ \\
\hline $12-13$ & 17 & 11 & $3.41(1.23-9.40)$ & 9 & $5.31(1.60-17.58)$ \\
\hline $14-17$ & 5 & 4 & $5.38(0.75-15.19)$ & 4 & $7.45(1.37-40.44)$ \\
\hline \multirow[t]{2}{*}{$18+$} & 1 & 1 & $5.15(0.29-90.44)$ & 1 & $10.32(0.56-191.14)$ \\
\hline & & & $p$-trend $=0.010$ & & $p$-trend $=0.002$ \\
\hline
\end{tabular}

*Adjusted for age, gender, smoking, alcohol, diabetes, hypertension, past history of colon polyps, BMI, triglyceride and fasting glucose.

**Adjusted for age, smoking, alcohol, diabetes, hypertension, past history of colon polyp, BMI, triglyceride, fasting glucose and HDL-C.

in those having a past history of colon polyps. Smoking was also found to be a risk factor for adenoma.

Homocysteine. Homocysteine levels did not correlate with the risk of colorectal adenoma development in the total population. Since male gender is itself a strong risk factor for adenoma and homocysteine levels in general were increased in males, we performed a subgroup analysis by gender to elucidate the exact association between homocysteine levels and adenomas. A higher plasma homocysteine concentration significantly increased the risk for adenoma and this trend was increased for advanced adenoma in the female population (Table 3 ).

\section{Discussion}

Many previous studies have investigated risk factors associated with colorectal adenoma and cancer. Recently, many studies support the idea that cardiovascular risk factors and colorectal cancer risk factors are clustered. ${ }^{(3-9)}$ Obesity, metabolic syndrome, hyperlipidemia, diabetes, and smoking are representative risk factors for cardiovascular disease as well as colorectal adenoma. ${ }^{(5-9)}$ The present results, in accordance with other studies, suggest that the well-known cardiovascular risk factors, old age and male sex, are also strong risk factors for colorectal adenoma. Using multiple logistic regression in the present study, high BMI, and low HDL$\mathrm{C}$ levels were found to be independent risk factors for adenoma and these risk factors are also known to be cardiovascular risk factors.

Homocysteine is a well-known biomarker for cardiovascular disease and more detailed clinical evaluations for cardiovascular disorders should be recommended for those with elevated plasma homocysteine concentrations. ${ }^{(13)}$ The plasma homocysteine concentration reflects the body's folate status. A diet including a consistently low consumption of vegetables results in a low body folate status as well as an elevated homocysteine concentration. ${ }^{(11)}$ Many epidemiologic studies support the contention that environmental risk factors, especially low vegetable consumption and high fat and meat consumption, are associated with colorectal cancer. ${ }^{(14)}$ Prospective studies in humans based on relatively large population samples have indicated that folate deficiency correlates well with the development of colorectal cancer as well as adenomas. ${ }^{(15-19)}$ Folate lies at the intersection of metabolic pathways that are involved in DNA methylation and biosynthesis. ${ }^{(15-19)}$ Decreased dietary folate intake markedly disturbs both DNA methylation and biosynthesis. ${ }^{(17-19)}$ It is difficult to evaluate the body's folate status based on serum folate levels because the serum folate level is strongly affected by ingested food and is sometimes elevated even in cases of folate deficiency. ${ }^{(12)}$ The plasma homocysteine concentration on the other hand, is inversely associated with the body's folate status and has the benefit of being reproducible. ${ }^{(12,13)}$ Higher homocysteine concentrations are known to be associated with colorectal cancer development through DNA hypomethylation as a result of an increase of sadenosyl methionine and faulty DNA synthesis due to decreased folate-derived cofactors. ${ }^{(17)}$ In the present study, we examined the association between the cardiovascular biomarker, homocysteine and colorectal adenoma. Plasma homocysteine concentration in 
the present study was measured by high performance liquid chromatography with a fluorescence detector which is the most popular method. ${ }^{(13)} \mathrm{We}$ found that males were more predominantly affected compared to females and male gender itself was a strong risk factors for colorectal adenoma. Hence, subgroup analysis by gender was undertaken to elucidate whether homocysteine itself can be a risk factor for adenoma. In subgroup analysis, the homocysteine level, i.e., low body folate status, did not affect development of adenoma in the male population; however, in the female population, high plasma homocysteine concentration was an independent risk factor for adenoma. We adjusted for age, gender, smoking, alcohol consumption, and diabetes, and excluded those with severe medical illness in an effort to determine whether high plasma homocysteine concentrations influenced the development of adenoma because plasma homocysteine concentration can be affected by age, gender, smoking, alcohol, drugs influencing folate metabolism, and concurrent illnesses. ${ }^{(12)}$

The susceptibility to develop adenoma under the influence of folate deficiency appears to differ by gender. Estradiol has been suggested to reduce adenoma or cancer in the colon. Previous large scale studies suggested the possibility that pronged supple-

\section{References}

1 Giovannucci E. Modifiable risk factors for colon cancer. Gastroenterol Clin North Am 2002; 31: 925-943.

2 Jänne PA, Mayer RJ. Chemoprevention of colorectal cancer. $N$ Engl J Med 2000; 342: 1960-1968.

$3 \mathrm{Kim} \mathrm{JH}$, Lim YJ, Kim YH, et al. Is metabolic syndrome a risk factor for colorectal adenoma? Cancer Epidemiol Biomarkers Prev 2007; 16: 15431546

4 Lim YJ, Kwack WG, Lee YS, Hahm KB, Kim YK. Increased pulse wave velocity reflecting arterial stiffness in patients with colorectal adenomas. $J$ Clin Biochem Nutr 2010; 47: 261-265.

5 Peipins LA, Sandler RS. Epidemiology of colorectal adenomas. Epidemiol Rev 1994; 16: 273-297.

6 Calle EE, Rodriguez C, Walker-Thurmond K, Thun MJ. Overweight, obesity, and mortality from cancer in a prospectively studied cohort of U.S. adults. $N$ Engl J Med 2003; 348: 1625-1638.

7 Ahmed RL, Schmitz KH, Anderson KE, Rosamond WD, Folsom AR. The metabolic syndrome and risk of incident colorectal cancer. Cancer 2006; 107: 28-36.

8 Nilsen TI, Vatten LJ. Prospective study of colorectal cancer risk and physical activity, diabetes, blood glucose and BMI: exploring the hyperinsulinaemia hypothesis. Br J Cancer 2001; 84: 417-422.

9 Frezza EE, Wachtel MS, Chiriva-Internati M. Influence of obesity on the risk of developing colon cancer. Gut 2006; 55: 285-291.

10 Lim YJ, Choi YH, Kim YH, et al. The association between plasma homocysteine concentration and the risk of rectosigmoid adenoma in heavy alcohol drinkers. Korean J Med 2004; 67: 475-479.

11 Jacques PF, Selhub J, Bostom AG, Wilson PW, Rosenberg IH. The effect of folic acid fortification on plasma folate and total homocysteine concentra- mentation with folate was protective against colorectal cancer with the greatest risk reduction among women. ${ }^{(20,21)}$ To date, an understanding of the pathophysiologic mechanisms responsible for this result has been lacking.

A limitation of the present study is the small female sample size. More studies based on a larger sample size should be performed to confirm that homocysteine, a cardiovascular biomarker of folate status, is indeed a risk factor for colorectal adenoma in women.

In conclusion, the well-known cardiovascular marker, homocysteine was found to be a meaningful risk factor for adenoma in the female population. This phenomenon was more prominent in cases of advanced adenoma. Hence, in those with elevated homocysteine concentrations, we would recommend the consumption of more vegetables to increase the body's folate status and eventually reduce the incidence of colorectal adenoma development. At the same time, screening colonoscopy should be recommended for females with high plasma homocysteine levels.

\section{Conflict of Interest}

No potential conflicts of interest were disclosed. tions. N Engl J Med 1999; 340: 1449-1454.

12 Boushey CJ, Beresford SA, Omenn GS, Motulsky AG. A quantitative assessment of plasma homocysteine as a risk factor for vascular disease. Probable benefits of increasing folic acid intakes. JAMA 1995; 274: 1049-1047.

13 Moghadasian MH, McManus BM, Frohlich JJ. Homocyst(e)ine and coronary artery disease. Clinical evidence and genetic and metabolic background. Arch Intern Med 1997; 157: 2299-2308.

14 Bingham S, Riboli E. Diet and cancer - the European prospective investigation into cancer and nutrition. Nat Rev Cancer 2004; 4: 206-215.

15 Lamprecht SA, Lipkin M. Chemoprevention of colon cancer by calcium, vitamin D and folate: molecular mechanisms. Nat Rev Cancer 2003; 3: 601614.

16 Boutron-Ruault MC, Senesse P, Faivre J, Couillault C, Belghiti C. Folate and alcohol intakes: related or independent roles in the adenoma-carcinoma sequence? Nutr Cancer 1996; 26: 337-346.

17 Martínez ME, Thompson P, Jacobs ET, et al. Dietary factors and biomarkers involved in the methylenetetrahydrofolate reductase genotype-colorectal adenoma pathway. Gastroenterology 2006; 131: 1706-1716.

18 Kim YI. Nutritional epigenetics: impact of folate deficiency on DNA methylation and colon cancer susceptibility. J Nutr 2005; 135: 2703-2709.

19 Ryan BM, Weir DG. Relevance of folate metabolism in the pathogenesis of colorectal cancer. J Lab Clin Med 2001; 138: 164-176.

20 Giovannucci E, Stampfer MJ, Colditz GA, et al. Multivitamin use, folate, and colon cancer in women in the Nurses' Health Study. Ann Intern Med 1998; 129: 517-524.

21 Fung T, Hu FB, Fuchs C, et al. Major dietary patterns and the risk of colorectal cancer in women. Arch Intern Med 2003; 163: 309-314. 